\title{
Internet contre Greta Thunberg : une étude discursive et argumentative
}

The Internet vs Greta Thunberg: a discursive and argumentative study

\section{Albin Wagener}

\section{OpenEdition}

\section{Journals}

Electronic version

URL: http://journals.openedition.org/aad/4747

DOI: $10.4000 /$ aad.4747

ISSN: 1565-8961

Publisher

Université de Tel-Aviv

\section{Electronic reference}

Albin Wagener, «Internet contre Greta Thunberg : une étude discursive et argumentative », Argumentation et Analyse du Discours [Online], 25 | 2020, Online since 15 October 2020, connection on 17 October 2020. URL : http://journals.openedition.org/aad/4747 ; DOI : https://doi.org/10.4000/aad. 4747

This text was automatically generated on 17 October 2020 .

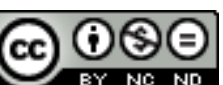

Argumentation \& analyse du discours est mis à disposition selon les termes de la licence Creative Commons Attribution - Pas d'Utilisation Commerciale - Pas de Modification 4.0 International. 


\title{
Internet contre Greta Thunberg : une étude discursive et argumentative
}

The Internet vs Greta Thunberg: a discursive and argumentative study

\author{
Albin Wagener
}

\section{Introduction}

1 Présentée comme l'égérie de la grève de l'école pour le climat (ou «Skolstrejk för klimatet » en suédois), mouvement repris par plusieurs collégiens et lycéens à travers le monde, Greta Thunberg s'est fait connaître à travers ses déclarations à la COP24 ou devant des assemblées, parlementaires ou non. En France, l'annonce de sa venue le 23 juillet 2019 à l'Assemblée Nationale, sur invitation du député non inscrit (ex-LREM ${ }^{1}$ ) Matthieu Orphelin, et accompagnée notamment de la climatologue Valérie MassonDelmotte, a polarisé à l'égard de la militante plusieurs critiques, commentaires et discours sceptiques. Dans les productions numériques médiatiques francophones (journaux, magazines, pureplayers, blogs, etc.), des auteurs ont décidé d'afficher publiquement leurs critiques. Qu'il s'agisse de Laurent Alexandre (fondateur du site internet doctissimo.fr ${ }^{2}$ ), de Michel Onfray ou encore de Pascal Bruckner, plusieurs personnalités et auteurs ont choisi de saisir leur clavier pour faire entendre leur point de vue. C'est dans ce contexte que nous proposons d'observer la manière dont les détracteurs de la militante suédoise argumentent au sein des discours qu'ils mobilisent et produisent, d'analyser les critiques qu'ils émettent, et de comprendre les logiques qui les sous-tendent.

\section{Une analyse discursive et argumentative de corpus}

2 Pour mieux comprendre le contexte de ces productions, et l'apex que celles-ci ont atteint à la fin du mois de juillet 2019, nous avons rassemblé un corpus comprenant 51 
productions écrites publiées sur des supports accessibles en ligne et facilement diffusables sur les réseaux sociaux (blogs, journaux en ligne, tribunes...) et s'échelonnant du 9 février, date de publication de l'article du magazine Reporterre concernant l'entourage relationnel de Greta Thunberg et l'affaire Rentzhog ${ }^{3}$, jusqu'au 30 juillet, soit une semaine après sa venue à l'Assemblée nationale et son discours devant les députés français. Nous avons choisi le 9 février comme date de départ, précisément parce que l'article rédigé par Isabelle Attard dans Reporterre ${ }^{4}$ a depuis été, sans doute malgré son auteure, relu et réinterprété par bon nombre de sites d'information libéraux, néo-conservateurs ou d'extrême-droite, nourrissant un nombre croissant de publications critiques conduisant à un moment discursif (Moirand 2007 : 4). Au sein de ce corpus, nous avons choisi de regrouper quatre types de sites, à savoir des journaux, des magazines, des pureplayers et des blogs. Ces sites sont accessibles en ligne lorsqu'on effectue une recherche concernant des critiques à l'égard de Thunberg sur Google ${ }^{5}$, y compris certains sites belges, suisses ou québécois, que nous avons intégrés, puisqu'ils participent de la sphère d'information francophone à laquelle ont accès les internautes en réponse aux mots-clés. Le corpus rassemble 50710 occurrences, 5434 formes linguistiques récurrentes et 2484 hapax (soit 4,90\% des occurrences). Pour une première analyse numérique du corpus, nous utilisons les logiciels Iramuteq et Wordsmith, pour des raisons que nous allons détailler ci-après, avant de compléter cette première approche par une étude argumentative systémique.

\subsection{Discours et argumentation : une proposition}

En premier lieu, il convient de définir ce que nous entendons par analyse du discours et en quoi cette analyse permet d'obtenir des résultats qui éclairent le fonctionnement des énoncés dans le corpus. A la fois concept et objet d'étude complexe et ambigu, le discours est constitué d'un ensemble de pratiques sociales mouvantes (Fairclough 1992) et influencé par les relations politiques entre individus et groupes d'individus (Dahlberg 2013 : 41). En ce sens, il permet une construction émergente du sens, en lien avec un environnement qui nécessite une adéquation de situation ${ }^{6}$ (Laks 1996: 109-110). Cette situation transforme la construction et la circulation du sens en un acte de cognition sociale (Bischofsberger 2002 : 167), structurant les sociétés et la circulation des identités (Howarth 2000), permettant ainsi la construction de prédiscours susceptibles de construire et d'activer des représentations (Paveau 2006). Une telle approche du discours ne peut faire l'impasse sur la notion d'interdiscursivité (Pêcheux 1975), soit le fait que les discours se construisent les uns les autres et par rapport aux autres, dans un univers d'une grande labilité (Garric et Capdevielle-Mougnibas 2009: 108). Ainsi positionnés, les discours représentent des ensembles qui présupposent des espaces doxastiques, permettant un partage et une communauté de croyance ou de connaissance (Stockinger 2001: 81). Pour ces raisons, nous utilisons la définition suivante pour parler de discours (Wagener 2019a : 39) :

Est appelé «discours » tout phénomène langagier (donc linguistique, mais pas exclusivement) qui a pour objet la construction, l'échange et la transformation d'un sens socialement situé et structurant, potentiellement signifiant politiquement, et nécessairement ancré dans une intersubjectivité essentielle, intentionnelle ou non, c'est-à-dire qui est produite et reçue par des sujets, qui peuvent s'exprimer de manière individuelle ou collective, à propos d'objets partageables. En ce sens, le discours n'est pas exclusivement linguistique, exclusivement communicationnel ou 
exclusivement social : il est à la fois au cœur et à la marge de ces dimensions, tout en les réunissant.

4 Dès lors, comment analyser les discours, et pourquoi proposer une analyse de corpus pour atteindre un but d'étude discursive? A la suite de Paul Baker, nous partons du principe que l'analyse de corpus expose l'effet incrémental du discours et la manière dont les associations linguistiques peuvent révéler l'émergence et la circulation des représentations (Baker $2006: 13)$ :

La linguistique de corpus, outre le fait qu'elle permette de réduire les biais d'interprétation, est une manière utile d'approcher l'analyse de discours, en raison de l'effet incrémental du discours. L'une des plus importantes façons de faire circuler et de renforcer les discours dans la société est l'utilisation du langage, et la mission des analystes du discours est de dévoiler la manière dont le langage est utilisé, souvent de manière plutôt subtile, afin de révéler les discours sous-jacents [...]. Ainsi, un simple mot, une simple phrase ou une simple construction grammaticale peuvent en eux-mêmes suggérer l'existence d'un discours [...]. Et c'est ici que les corpus deviennent utiles. Une association entre deux mots, qui se produit de manière répétitive dans le langage naturel, est une preuve bien plus pertinente pour un discours hégémonique sous-jacent, rendue explicite par la collocation lexicale, qu'un simple cas (notre traduction).

5 Comme nous le verrons avec le logiciel Wordsmith, la position de Baker prend tout son sens au moment de l'analyse des co-occurrences lexicales: cette phase permet de proposer une cartographie systémique de la manière dont les lexèmes sont associés dans le corpus, comme cela a par ailleurs déjà été travaillé dans l'AD dite à la française (Tournier 1985, Reinert 1990, Marchand 1998).

6 Toutefois, ces précisions nécessitent d'y associer une définition de l'argumentation et de l'analyse argumentative. A ce titre, nous souhaitons nous appuyer sur les travaux de Ruth Amossy, qui invitent à considérer l'argumentation comme un phénomène logé au cœur du fonctionnement discursif (Amossy 2011 : 16) :

l'argumentation (1) est définie comme un acte de communication où l'auditoire est primordial, et doit toujours être pris en compte ; [...] (2) se fait à travers la langue naturelle, dont elle mobilise toutes les ressources ; (3) se manifeste aussi bien sous forme de discours à visée de persuasion, que dans la dimension argumentative d'un discours qui n'entreprend pas expressément de persuader ; [...] (4) comporte un dosage variable d'appel à la raison et de recours au sentiment. [...] En bref, l'argumentation comme façon d'influer sur des façons de voir et de penser est une dimension constitutive du discours, au même titre que l'énonciation, le dialogisme ou la construction d'une image de soi (ethos). Elle est au cœur du fonctionnement discursif, dont il revient à l'analyste de dégager les modalités complexes.

7 Ainsi l'argumentation, qu'Oswald Ducrot avait défini comme «l'acte linguistique fondamental »(Ducrot 1980: 11), permet une analyse située au sein des espaces démocratiques, afin de distinguer les procédés argumentatifs et leurs caractéristiques (Nicolas 2015). Toutefois, une analyse des procédés argumentatifs à l'intérieur du corpus ne consiste pas en une réification métrique par application théorique, mais bien en une compréhension des logiques argumentatives à l'œuvre dans le corpus, pour ce qu'il est, et par rapport au contexte qui l'a généré (Amossy 2015) :

Tout d'abord, aucune " application » de la théorie aux textes n'est souhaitable, ni même possible. C'est qu'il ne s'agit pas de recettes d'analyse, de catégories préfabriquées qu'il suffit de retrouver dans les textes. Chaque discours concret, ou ensemble de discours, se construit en imbriquant d'une façon singulière les éléments discursifs, les figures, les types d'arguments qu'il sélectionne, et c'est de cette réélaboration souvent complexe que se dégage son sens, sa logique et sa 
finalité. Dans l'analyse des données, il ne s'agit donc pas d'étiqueter et de cataloguer, mais de faire voir des configurations et de montrer ce qui s'y construit. l'analyse discursive et argumentative que nous proposons doit permettre de comprendre les logiques qui animent les critiques proférées contre Greta Thunberg dans une logique d'analyse située de l'argumentation (Doury et Plantin 2015).

\subsection{Analyse systémique du discours}

Notre méthode d'analyse se veut systémique, afin de dégager les structures représentationnelles du corpus. Dans ce cadre, ces structures sont concentrées autour de la construction du personnage de Greta Thunberg entreprise par ses détracteurs, sur le modèle de l'analyse systémique de discours (Wagener 2017) et de son application aux spécificités des espaces numériques (Wagener 2019b). Cette analyse systémique du discours se focalise sur le repérage de nœuds sémantiques, soit les ensembles de cooccurrences lexicales particulièrement signifiantes qui, reliés entre eux, forment une cartographie des représentations (Wagener 2019a: 112-113). Cette méthodologie nécessite les étapes suivantes :

- Une présentation des analyses des catégorisations sémantiques, proposées par Iramuteq ${ }^{7}$, afin de déterminer une première représentation $\mathrm{du}$ corpus et des positionnements énonciatifs exprimés dans les textes ;

- Suite à cette étape, il convient de retourner au corpus en proposant un comptage lexicométrique de co-occurrences (ici produit par le logiciel Wordsmith), grâce auquel il est possible de proposer un ensemble de nœuds sémantiques à haute fréquence ;

- Pour terminer, l'ensemble de ces données doit être croisé avec des éléments qui échappent au principe de haute fréquence, mais donnent néanmoins des informations sur les logiques d'argumentation et de signification.

Le but est d'utiliser l'approche lexicométrique, non pas dans une optique de comptage numérique qui ne donnerait aucune indication sur les processus de signification, mais de faire émerger des espaces représentationnels (Bendinelli $2011: 110)$ :

Les espaces représentationnels donnent une clef de lecture originale aux prises de parole qui les sous-tendent, car ils permettent de 'voir' le monde que le locuteur construit et les relations qu'il entretient avec les éléments qui le composent. Chemin faisant, ils offrent la possibilité d'identifier une partie des connaissances convoquées lors de l'interprétation ${ }^{8}$. [...] La figure obtenue reconstruit un prototype des productions prises en compte, elle en subsume les éléments les plus représentatifs, indépendamment des variations thématiques.

11 L'approche élaborée par Marion Bendinelli, qui s'inspire sans équivoque des travaux de Paul Chilton (2005), met en lumière une topographie discursive qui complète l'analyse des procédés argumentatifs que nous isolons au sein des textes du corpus. Dans le contexte d'une étude discursive et argumentative de corpus, cette méthode semble permettre de répondre à la question des argumentaires à l'œuvre dans les propos des opposants à Greta Thunberg. Il ne s'agit évidemment pas (et il est utile de le souligner) de qualifier l'action de Thunberg ou les méthodes de communication qu'elle et son entourage utilisent, mais de se focaliser sur les critiques qu'elle provoque par son action. 


\section{Etude du corpus}

12 Il est utile de rappeler la constitution du corpus, afin d'aborder son étude avec précision,. Les 51 textes réunis au sein du corpus sont tous issus de médias numériques, repérés par Google en précisant les mots-clés « critique Greta Thunberg »:

- 17 textes publiés dans des journaux (articles et tribunes: Valeurs Actuelles, Le Devoir, Le JDD, Le Figaro, RTBF, 20 Minutes, Le Monde, Libé) ;

- 15 articles publiés dans des pureplayers9 (Atlantico, Boulevard Voltaire, Huffington Post, AgoraVox, Reporterre...) ;

- 11 textes publiés dans des magazines (Elle, Causeur, Marianne, Le Point...) ;

- 7 textes de blog (celui de Michel Onfray, Minurne Résistance, Les 4 Vérités);

- 1 pétition publiée sur Change.org.

Les métadonnées utilisées pour coder le corpus permettent d'isoler la source du texte, la date de publication et le pays de provenance. Ces textes sont publiés entre le 9 février et le 30 juillet, avec un pic de publication le 23 juillet (10 publications, soit un cinquième du corpus), au moment de la visite de Thunberg à l'Assemblée nationale française. 44 textes proviennent d'acteurs de France, trois du Canada, trois de Belgique et un de Suisse. Nous avons choisi de rassembler des textes, avec une concentration au niveau des textes issus de journaux et de pureplayers, suivis par les magazines et les blogs, parce que ces résultats sont représentatifs des résultats obtenus via Google lorsqu'il s'agit pour l'internaute de chercher des productions critiques envers la militante suédoise. Ici, l'écrasante majorité des textes, sauf ceux publié dans Reporterre, Marianne et Media Presse Infos, est signée par des hommes (soit 48 sur 51). 


\subsection{Classification des réseaux sémantiques du corpus}

\subsubsection{Faire émerger une classification des réseaux lexico-sémantiques avec le logiciel Iramuteq (méthode Alceste) ${ }^{10}$}
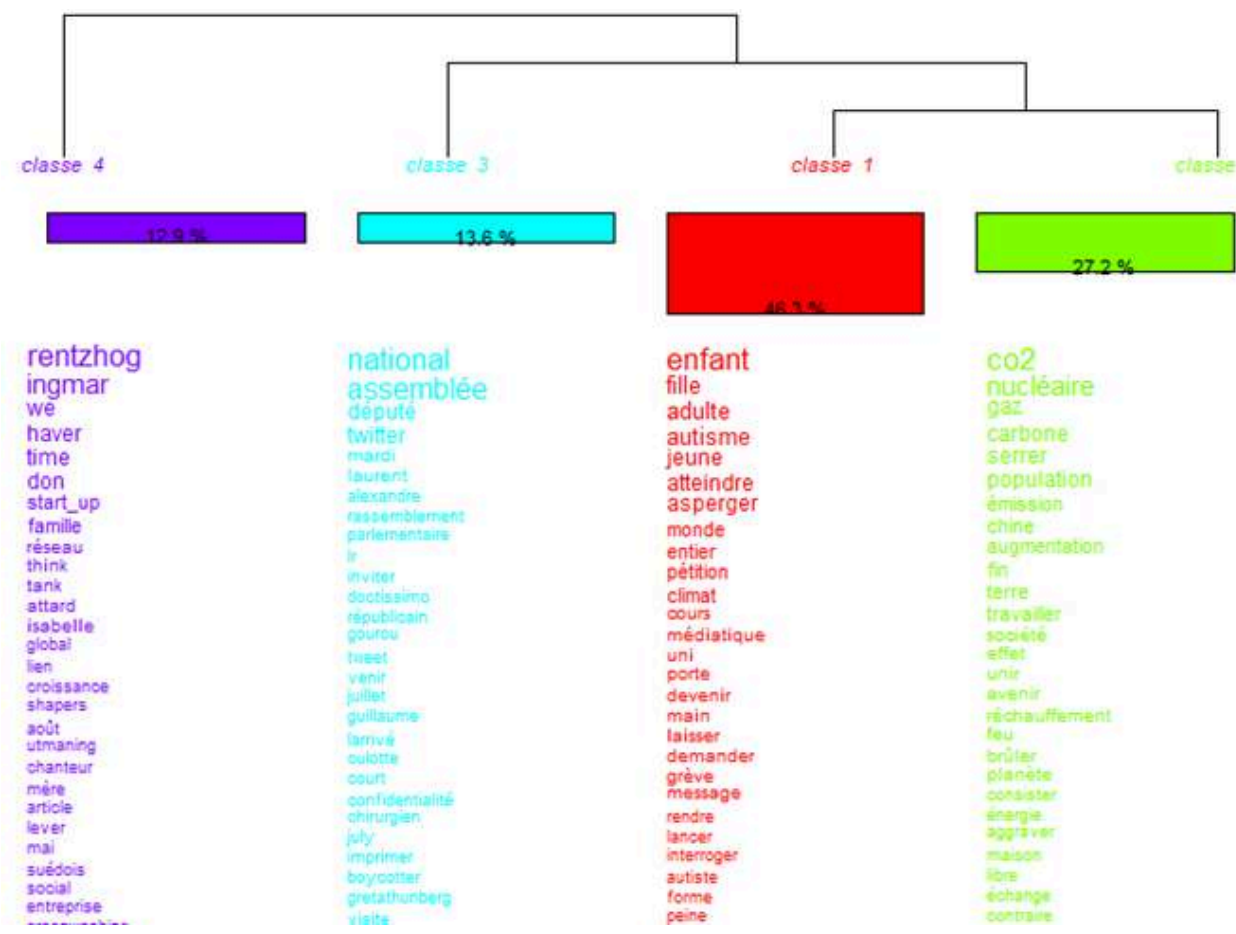

Fig. 1. Dendrogramme du corpus GT (extrait produit par le logiciel)

Cette perspective permet d'isoler quatre classes concernant la logique de sens à l'œuvre au sein des critiques à l'égard de Thunberg :

- la première classe (classe $4,12.9 \%$ du corpus), ou classe racine (soit celle dont vont découler les autres classes) se consacre à l'affaire Igmar Rentzhog, révélée en France par le magazine Reporterre, sur base de l'enquête du journaliste suédois Andreas Henriksson - enquête qui avait entraîné un droit de réponse de Greta Thunberg, puis une contre-enquête du journaliste lui-même ;

- la seconde classe (classe 3, 13.6\% du corpus) polarise les critiques autour du contexte de la venue de Greta Thunberg à l'Assemblée nationale française, ce qui confirme l'effet de polarisation des critiques en lien avec cet événement de la vie politique française ;

- la troisième classe (classe 1, 46,3\% du corpus), de loin la plus fournie, est entièrement consacrée à Thunberg, dans un florilège de comparaisons, de métaphores et de jugements de valeurs qui cantonnent la militante à sa jeunesse, sa féminité et au syndrome d'Asperger ;

- la quatrième classe (classe 2, 27,2\% du corpus) se polarise sur les questions écologiques et politiques, ce qui indique que les critiques concernant Thunberg ne concerneraient pas exclusivement sa personne, mais également ses prises de position.

Ainsi émerge une première représentation générique du corpus, qui nécessite d'être rapprochée des formes énonciatives. Pour comprendre la répartition de ces classes sémantiques, il faut compléter cette étude par l'analyse factorielle de correspondances ${ }^{11}$, qui révèle le paysage sémantique, en fonction d'éloignement des classes et de la manière dont les textes y contribuent. 


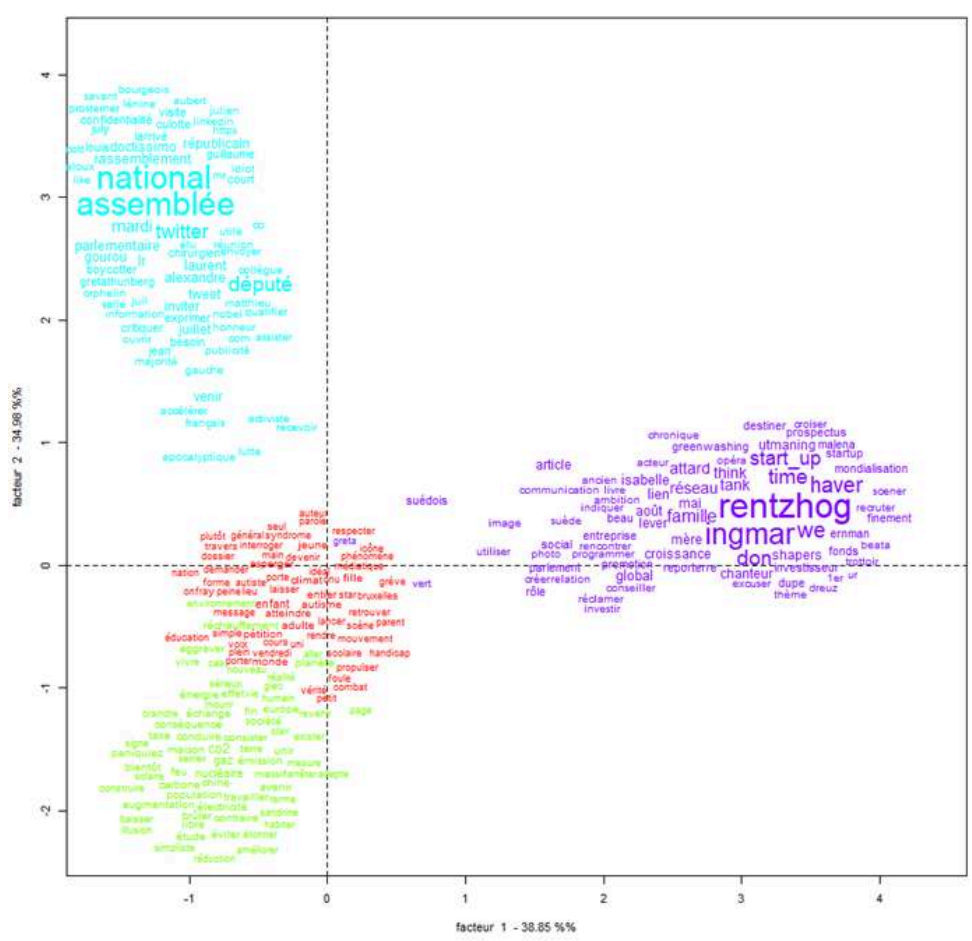

Fig. 2. Analyse factorielle de correspondances du corpus GT

Cette perspective montre que la classe 4, qui cristallise la théorie de l'affaire Ingmar Rentzhog, reste éloignée de deux ensembles qui s'opposent: d'un côté (en haut à gauche) la classe 3, qui concerne la venue de Thunberg à l'Assemblée nationale française; de l'autre les classes 1 et 2, dont la relative proximité montre que le ciblage des critiques concerne à la fois Thunberg et ses positions écologiques. Il est à noter ici que la classe 1, qui concerne Thunberg, se retrouve au centre des axes du graphe, ce qui tend à démontrer que c'est bien ici sa personne qui polarise les critiques; dans le même temps l'analyse factorielle oppose la classe 3 (visite à l'Assemblée nationale) et la classe 2 (positionnement écologique), segmentant ainsi l'événement de la visite lui-même des questions politiques, qui ne seraient donc pas utilisées par les opposants à sa venue à l'assemblée. Une hypothèse peut alors être formulée : si les critiques de Greta Thunberg se sont opposés à sa venue à l'Assemblée nationale, c'est moins pour ses idées écologistes que pour elle-même. Cette hypothèse doit être vérifiée au cours d'une analyse des énoncés. 


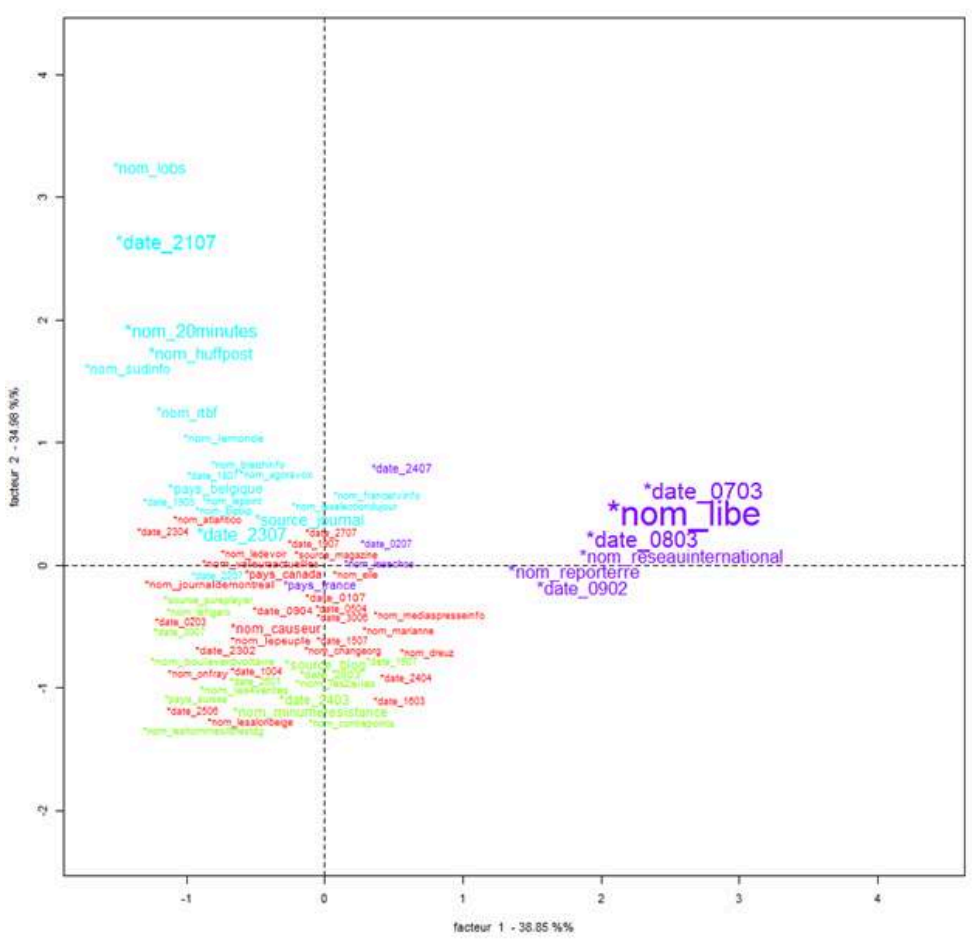

Fig. 3. Analyse factorielle de correspondances des sources du corpus GT

Dernière figure, l'analyse factorielle de correspondances aide à montrer la manière dont blogs, magazines, pureplayers et journaux se positionnent. On remarque par exemple que l'affaire Rentzhog (classe 4) semble en priorité traitée par Libé et Reporterre (ce qui est parfaitement normal pour cette seconde source), et que les articles qui ciblent Thunberg en tant que personne sont à trouver dans Atlantico, Elle, Causeur, Marianne et un ensemble de blogs et pureplayers appartenant à la mouvance de droite néo-conservatrice ou d'extrême-droite. Il s'agit ici de points forts soulignés par Iramuteq, ce qui ne veut pas dire que l'exclusivité de ces contre-argumentations repose seulement sur ces sources et qu'elles ne traitent d'aucun autre sujet.

\subsubsection{Analyse lexicométrique des co-occurrences lexicales et énoncés correspondants}

Pour mettre en œuvre cette analyse lexicométrique et présenter les formes les plus fréquentes de co-occurrences lexicales, nous utilisons le logiciel Wordsmith (Scott 2001), qui permet d'obtenir une vue directe des occurrences les plus notables. Pour commencer, nous sélectionnons le lexème "Greta", parfois utilisé sans le nom de famille «Thunberg» (ce qui mériterait en soi une analyse propre), et qui permet de relever d'ores et déjà le nombre de co-occurrences signifiantes en fréquences absolues.

\begin{tabular}{|l|l|}
\hline Collocate List & \\
\hline Word & Total \\
\hline GRETA & 545 \\
\hline
\end{tabular}




\begin{tabular}{|c|c|}
\hline THUNBERG & 360 \\
\hline JEUNE & 40 \\
\hline SUÉDOISE & 27 \\
\hline GRÈVE & 20 \\
\hline ASSEMBLÉE & 15 \\
\hline ELLE & 14 \\
\hline ACTIVISTE & 13 \\
\hline MILITANTE & 11 \\
\hline JEUNES & 10 \\
\hline CLIMAT & 10 \\
\hline CLIMATIQUE & 9 \\
\hline RENTZHOG & 9 \\
\hline LIVRE & 9 \\
\hline PRODUIT & 9 \\
\hline DISCOURS & 9 \\
\hline PARLEMENT & 9 \\
\hline ÉGÉRIE & 9 \\
\hline INGMAR & 8 \\
\hline PHÉNOMÈNE & 8 \\
\hline NATIONALE & 8 \\
\hline PARENTS & 8 \\
\hline FAMILLE & 8 \\
\hline SAINTE & 7 \\
\hline ICÔNE & 7 \\
\hline TRAIN & 7 \\
\hline MÈRE & 7 \\
\hline MÉDIATIQUE & 6 \\
\hline
\end{tabular}




\begin{tabular}{|l|l|}
\hline SUCCÈS & 6 \\
\hline BEATA & 6 \\
\hline AGGRAVERAIT & 6 \\
\hline RÉCHAUFFEMENT & 6 \\
\hline FABRIQUÉ & 6 \\
\hline ÉCOLO & 6 \\
\hline HISTOIRE & 6 \\
\hline PHOTO & 6 \\
\hline PROTÉGER & 6 \\
\hline
\end{tabular}

Fig. 4. Co-occurrences lexicales du lexème « Greta »

Ici, il est question de l'affaire Ingmar Rentzhog, des relations avec la famille de Greta, d'un traitement "sacré» de la militante suédoise ou supposé tel, avec des termes comme " égérie ", « sainte » ou « icône » (le retour au corpus permet de constater qu'il s'agit d'une utilisation sarcastique de ces termes), et de la notion d'artificialité (« produit » et «fabriqué »). Jusqu'ici la neutralité de la métrique montre ici ses limites et illustre bien le fait qu'une approche purement numérique ou automatisée de l'analyse linguistique, sémantique ou discursive ne saurait permettre de comprendre les représentations signifiantes contenues dans les énoncés.

Pour mieux comprendre l'intérêt d'une telle analyse, il convient de comprendre la manière dont ces lexèmes de co-occurrence première (ici lexèmes $\mathrm{A}$ ) interagissent avec leur environnement propre (ou lexèmes $B$ ), ce qui permettra de dégager des nœuds sémantiques (Wagener 2017, Wagener 2019a) qui requalifieront ensuite Thunberg, à la manière d'un réseau de poly-co-occurrences (Martinez 2012). En d'autres termes, si nous isolons par exemple le lexème " jeunes » et que nous remarquons que celui-ci est utilisé dans des contexte négatifs, alors nous pourrons vraisemblablement, par contamination, déduire que le lexème "Greta ", lorsqu'il est cité avec le lexème A "jeune", porte l'ensemble des valeurs axiologiques négatives contenues dans la fabrication d'énoncés comprenant des lexèmes $B$.

\begin{tabular}{|l|l|}
\hline Collocate List & \\
\hline Word & Total \\
\hline JEUNE & 159 \\
\hline FILLE & 47 \\
\hline GRETA & 43 \\
\hline SUÉDOISE & 43 \\
\hline
\end{tabular}




\begin{tabular}{|l|l|}
\hline THUNBERG & 31 \\
\hline 16 & 15 \\
\hline ANS & 14 \\
\hline MILITANTE & 12 \\
\hline ACTIVISTE & 12 \\
\hline FEMME & 11 \\
\hline ÉCOLOGISTE & 10 \\
\hline ÂGE & 5 \\
\hline PROGRAMMÉ & 5 \\
\hline TRANSFORMER & 5 \\
\hline HÉROÏNE & 5 \\
\hline INTERNATIONALE & 5 \\
\hline DERRIÈRE & 5 \\
\hline IMAGE & 4 \\
\hline CLIMAT & 4 \\
\hline AUTISTE & 4 \\
\hline CLIMATIRIE & 4 \\
\hline ASSEMBLÉE & 4 \\
\hline
\end{tabular}

Fig. 5. Co-occurrences lexicales du lexème « jeune »

21 Avec « jeune », l'authenticité et la responsabilité de la personne publique sont mises en doute, avec des lexèmes comme "programmé ", «transformer », " derrière » (au sens des forces qui se cacheraient derrière Greta) ou encore « image ». Plus spécifiquement, les exemples suivants sont à noter :

-1. Succès garanti tant la nouvelle star est jeune

-2. Comment expliquer ce choix, et faire comprendre à la jeune fille que l'école est une arme, non un frein

-3. C'est une jeune fille au corps neutre et à la parole belliqueuse

-4. L'endoctrinement médiatique commence dès le plus jeune âge

-5. Tout a été programmé pour transformer la jeune Greta en héroïne internationale 
exemples donnent à voir la manière dont le lexème «jeune » apparaît au sein du corpus. Avec ce qualificatif, ce ne sont pas les positionnements politiques de Thunberg qui sont visés, mais le fait que son âge semble être, par sa nature même, un disqualifiant concernant son discours. Pour approfondir cette logique, nous proposons des exemples liés au lexème "enfants ", également utilisé, et qui confère un degré supplémentaire de dénigrement de la parole de la militante :

-6. Mettre en avant des enfants présentant une souffrance est répugnant

- 7. Ce n'est pas aux enfants de faire de la politique

- 8. Notre époque voit arriver au-devant de la scène des enfants rois

-9. Bientôt, on ira à la maternelle demander l'avis des enfants sur l'avenir de la planète

-10. Au risque de déplaire, je dirai que la place des enfants et des adolescents n'est pas dans la rue, mais à l'école

La manière dont le lexème " enfants » est mis en scène dans les discours des anti-Greta Thunberg laisse peu de place au doute quant à leur représentation de l'enfance : tour à tour manipulés ou pas à leur place, les enfants seraient sommés de laisser les adultes opérer une politique responsable. Au passage, il convient ici de faire remarquer que Thunberg a seize ans en 2019, au moment de ces publications, et que l'on pourrait plutôt parler d'une adolescente que d'une enfant, qui n'est qu'à deux ans de la majorité. Nous formulons l'hypothèse que si le lexème " enfants » est utilisé aussi abondamment dans le corpus, c'est notamment dû à l'apparence physique de Greta Thunberg ou à son look, qui est par ailleurs également assigné puis utilisé par ses détracteurs.

Rappelons au passage que l'argument de l'âge n'avait, par exemple, jamais été opposé à Malala Yousafzai lorsque celle-ci a obtenu le prix Nobel de la paix à l'âge de 17 ans, et qu'il se pourrait par ailleurs que l'argument de la jeunesse aille de pair avec l'argument du genre. Puisqu'il s'agit en effet d'une «jeune fille» ou d'une "jeune femme» (en fonction des énonciateurs), il parait intéressant de contextualiser la manière dont les lexèmes « fille » et « femme » sont présentés au sein du corpus :

-11. Une jeune femme déconcertante, peu expressive, voire apathique

-12. La jeune femme de 16 ans devrait recevoir une fessée

-13. Il n'y a plus moyen de critiquer cette jeune femme sans risquer de passer pour un climatosceptique

-14. Mais c'est une jeune fille! Avec des tresses, comme Fifi Brindacier

-15. On dirait la petite fille aux allumettes du conte d'Andersen

Outre les références stylistiques au patrimoine culturel suédois («Fifi Brindacier» ou «la petite fille aux allumettes»), il est aisé de remarquer que "femme» se retrouve quasi systématiquement accolé à " jeune », et que cette jeunesse assortie de la féminité semble indiquer que Thunberg ne serait en fait pas maitresse de ses déclarations ou de son combat, mais plutôt un être manipulé par son entourage. En outre, son comportement est également souligné par ses détracteurs, ce qui semble indiquer que son handicap pourrait être utilisé comme un argument :

-16. Autiste Asperger, Greta Thunberg est dépressive, végane

-17. Se servir de la frimousse d'un enfant autiste comme tête de gondole est une idée osée mais imparable

-8. En révélant qu'elle est atteinte d'un autisme Asperger, ses parents en ont fait un bouclier humain

-9. Greta Thunberg affiche son Asperger comme un titre de noblesse 
-10. Derrière cette jeune autiste, affectée du syndrome d'Asperger, se cachent des élites écologistes

Outre la jeunesse et la féminité, le syndrome d'Asperger parachève de noircir le tableau d'une personne fragile, manipulée par son entourage, toujours dans une logique de mise en doute de l'authenticité d'une personne, qui constituerait une pure production communicationnelle.

\section{Argumentaires et formes énonciatives}

Pour comprendre la manière dont discours et argumentations se construisent, il est important d'opérer un retour aux énoncés afin d'en saisir les argumentaires (Amossy 2017). Après étude du corpus, nous isolons les argumentaires suivants :

- La fragilité comme vecteur de délégitimation

- L'invalidité d'une parole non experte

- L'appel à l'émotion et la parole non rationnelle

- La conspiration du greenwashing

- La sacralité mystique ou l'impossibilité du dialogue

- L'apparence physique comme ultime repoussoir

Afin d'expliciter ce que nous entendons par ces six argumentaires contenus dans les discours, nous en détaillons la structure ci-après.

\subsection{La fragilité comme vecteur de délégitimation}

Comme nous l'avons souligné grâce aux exemples liés à la jeunesse, à l'enfance, à l'autisme Asperger ou encore à la condition de genre de jeune fille ou de jeune femme, les critiques produites à l'encontre de Greta Thunberg se positionnent par rapport à la fragilité supposée de la militante ou des jeunes qui la suivraient lors des grèves scolaires du vendredi. Sa fragilité inhérente l'empêcherait donc d'être en possession de ses moyens ou de ses productions discursives, une fragilité qui alimente par ailleurs la théorie de la conspiration du greenwashing. Ainsi, les détracteurs de Thunberg utilisent l'inquiétude (sincère ou feinte) qu'ils ressentiraient à l'encontre de la militante suédoise, afin de la transformer en victime et d'alimenter la thèse de son irresponsabilité :

- Greta Thunberg évolue dans un milieu d'extrême-gauche avéré où règne une idéologie qui lui fait perdre tout discernement [...] c'est une enfant complètement manipulable et donc manipulée (Gregory Roose, Agoravox, 27 juillet 2019) ;

- Ce n'est pas aux enfants de faire de la politique et les parents de Greta Thunberg sont irresponsables de mettre en avant une enfant si fragile et qui a besoin de repos et de calme (Laurent Alexandre, Le Point, 23 juillet 2019);

- Son handicap la rend très facilement manipulable, c'est la marionnette idéale et de surcroît pas trop chère pour ses employeurs (Marc Le Stahler, Minurne Résistance, 24 mars 2019).

Fragile en raison de sa famille ou du milieu dans lequel elle évolue, fragile parce que trop jeune ou parce qu'atteinte d'une forme d'autisme, Thunberg ne serait donc pas une militante combative portée par une authentique sincérité, mais une marionnette tombée entre les mains de personnes malfaisantes, ce qui en ferait une victime qui vivrait un calvaire, et ce au grand jour. Se focaliser sur cette manipulabilité de 
l'énonciatrice rend de facto inopérant son discours, puisque ce discours ne serait pas le sien et que l'énonciatrice ne se rendrait pas compte de ce qu'elle dit.

\subsection{L'invalidité d'une parole non experte}

31 Greta Thunberg n'est pas une scientifique, ni une élue politique : en tant que militante, elle est réduite par ses détracteurs à un rôle d'activiste qui empêcherait les experts de faire leur travail. N'ayant pas été élue ou co-rédactrice des rapports du GIEC, ses discours seraient donc inaudibles et ne permettraient pas de faire avancer de manière intelligente les choix politiques concernant les changements climatiques :

- Pour lutter intelligemment contre le réchauffement climatique, nous n'avons pas besoin de gourous apocalyptiques, mais de progrès scientifique et de courage politique (tweet de Guillaume Larrivé du 20 juillet 2019 repris par Anthony Bertelier dans Huffpost, 21 juillet 2019) ;

- Et le messager, la messagère pour la circonstance, n'est pas symboliquement le meilleur vecteur pour une politique qui mérite sans doute mieux qu'un cliché in fine assez « malaisant » (Arnaud Benedetti, Valeurs Actuelles, 22 juillet 2019);

-L'écologie est un sujet trop sérieux pour être confié à des enfants perturbés. [...] Laissons parler les grandes personnes, celles qui ont étudié la question et qui proposent des solutions, pas des jérémiades (Jérôme Godefroy, Medium, 30 juin 2019).

Peu importe que l'espace démocratique permette à chacun de s'exprimer, y compris, d'ailleurs, à des auteurs, philosophes ou bloggeurs qui ne sont pas plus spécialistes du climat que Greta Thunberg. Ici, le manque d'expertise supposé de l'énonciatrice invaliderait de manière inconditionnelle l'ensemble de son discours, peu importe qu'elle prenne la peine de se documenter et de citer des pages du rapport du GIEC et d'y renvoyer régulièrement son auditoire. Ceci montre la manière dont les opposants à l'action de Thunberg considèrent une parole qui ne serait ni politique, ni experte; une telle dévalorisation du discours d'autrui sur la seule foi d'une absence de doctorat ou d'engagement politique (au sein d'un parti, en tout cas, et non pas dans un mouvement qui ne s'inscrit pas dans la vie politique traditionnelle) est relativement éloquente.

\subsection{L'appel à l'émotion et la parole non rationnelle}

Selon ses détracteurs, les formules rhétoriques de Greta Thunberg procèderaient uniquement par références émotionnelles ou sentimentales, empêchant toute discussion rationnelle concernant le changement climatique. Ce faisant, ses propos prendraient une place trop importante dans l'espace médiatique, privant les destinataires d'échanges scientifiques :

- On crée des générations d'enfants terrifiés par notre propagande, et, ce faisant, on leur vole leur enfance et leur insouciance. On les mobilise moins qu'on ne les tétanise (Pascal Bruckner, Le Figaro, 10 avril 2019);

- Le ressort de leur action a plus à voir avec l'émotion qu'avec la raison, avec la religion qu'avec la science (Christian Rioux, Le Devoir, 19 juillet 2019) ;

- Un phénomène de contagion par lequel une idée dominante se répand facilement dans les esprits galvanisés par l'émotion commune. La pensée collective devient alors totalement subjective (Michel Janva, Le Salon Beige, 22 juillet 2019). 
Deux auteurs se distinguent, à savoir Michel Onfray (sur son blog) et John Goetelen (sur son blog hébergé par la Tribune de Genève), qui reprochent au contraire à l'impétrante son absence supposément totale d'émotion. Il ne lui est pas reproché, en tant que jeune femme, d'être elle-même en proie à des émotions, mais bien de les susciter auprès de son auditoire. S'il est vrai que Greta Thunberg est coutumière des formules choc et des phrases réutilisables dans la sphère médiatique, c'est précisément parce que son rôle d'activiste est de marquer l'espace public en utilisant un discours porteur de slogans afin de sensibiliser à une cause. Sensibiliser n'est pas informer, mais susciter le désir de s'informer; en confondant deux actes de langage, voire deux registres énonciatifs, ses détracteurs semblent nier l'utilité des militants et leur rôle essentiel dans la sensibilisation à des causes humaines ou des luttes sociales, comme cela a pourtant déjà été le cas par le passé. Rosa Parks n'était, par exemple, pas docteure en sciences politiques, mais son action a pourtant participé de manière positive aux combats menés par la communauté noire aux États-Unis.

\subsection{La conspiration du greenwashing}

L'affaire Ingmar Rentzhog a contribué à jeter le trouble sur la sincérité et l'authenticité de l'activisme de la militante suédoise, en mettant en lumière les relations familiales, amicales et professionnelles qui semblaient se dissimuler derrière l'image de Thunberg telle que véhiculée dans les médias. En France, c'est l'éco-anarchiste autoproclamée Isabelle Attard (une des rares auteures féminines du corpus) qui relaie, via Reporterre, l'enquête du journaliste suédois Andreas Henriksson. Si l'enquête permet de lever le voile sur l'entourage de la militante suédoise, et qu'Isabelle Attard rappelle que le combat de Greta Thunberg est juste, le mal et fait - très vite, une impression de conspiration prend le relais :

-Et de l'autre, nous avons une famille de milliardaires comptant une ex-ministre qui investit dans cette start-up, puis qui embauche Ingmar Rentzhog dans un think tank développant les thèmes de la croissance verte, de l'économie circulaire, bref, de greenwashing (Isabelle Attard, Reporterre, 9 février 2019);

- Mais aujourd'hui, cette belle image se fissure légèrement. Certains lui reprochent d'être une 'marionnette marketing' manipulée par Ingmar Rentzhog, un start-upper suédois pro du « greenwashing » (Patrick Williams, Elle, 5 avril 2019);

- Avant d'engager une réflexion sur le concept de pédo-instrumentalisation, nous donnons des détails sur l'origine des "grèves de la jeunesse pour le climat». Elles ne sont en rien spontanées. Il apparaît que son instigatrice suédoise de 16 ans, Greta Thunberg, est à la base d'une construction médiatique à vocation financière (Stanislas de Larminat, Les 2 Ailes, 28 mars 2019).

Peu importe que Thunberg réponde sur ces points ou que Rentzhog lui-même intervienne pour expliciter ce qu'il qualifie de malentendu, le mal est fait : bon nombre d'auteurs néo-conservateurs ou de sites d'extrême-droite vont reprendre les propos d'Attard pour utiliser les ressorts rhétoriques, argumentatifs et discursifs du complot (cf. Nicolas 2014). 


\subsection{La sacralité mystique ou l'impossibilité du dialogue}

37 De façon sarcastique, ceux qui profèrent un discours critique à l'égard de Thunberg invoquent régulièrement des figures liées au sacré ou au mystique pour décrire la militante. Il s'agit peut-être de la logique argumentative la plus intéressante, tant elle emprunte à des schèmes argumentatifs relativement traditionnels des énonciateurs masculins :

- Il y a en effet quelque chose de mystique dans ce regard virginal et cette façon simple de dire les choses. Ceux qui l'ont approchée semblent évoquer une forme de magnétisme, comme s'ils avaient été touchés par la grâce. D’ailleurs, Greta Thunberg ne nous annonce-t-elle pas l'Apocalypse, comme tant de jeunes filles inspirées avant elle, de Thérèse de Lisieux à Bernadette Soubirous? (Christian Rioux, Le Devoir, 19 juillet 2019) ;

- Le réchauffisme préfère maintenant s'appuyer sur les miracles de Sainte Greta. Il a raison : c'est plus sûr. Une tournée d'apparitions de Sainte Greta fait plus pour la cause que deux ou trois COPs (Rémy Prudhomme, Contrepoints, 7 mai 2019);

- La jeune fille au regard d'azur, conjuguant l'indignation de Jésus chassant les marchands du Temple et la pureté farouche d'Athéna (Olivier Babeau, Le Figaro, 26 février 2019).

Greta Thunberg se retrouve assimilée à une figure de proue mystique, considérée comme sacrée par la foule qui la suivrait et dont la nature prophétique, annonciatrice d'apocalypse, n'aurait plus rien à voir avec le traitement rationnel du changement climatique, mais avec une religion à laquelle il s'agirait de se convertir. Au-delà de cette fabrication de figure sacrée, qui s'apparente à une stratégie de l'homme de paille qui auto-censurerait de facto toute possibilité de dialogue, on retrouve ici les stratégies représentationnelles qui attribuent aux femmes des caractéristiques proches de la sorcellerie (Nathan, Kelkar et Xiaogang 1998).

\subsection{L'apparence physique comme ultime repoussoir}

Si cet ensemble d'argumentaires ne suffisait pas à convaincre du fait que Greta Thunberg soit l'icône dangereuse d'une manipulation de masse, il reste le rempart des attaques ad feminem, à savoir le physique (Frigan et Kérisit 2000). Ici, certains auteurs s'en donnent à cœur joie :

- Cette jeune fille arbore un visage de cyborg qui ignore l'émotion - ni sourire ni rire, ni étonnement ni stupéfaction, ni peine ni joie. Elle fait songer à ces poupées en silicone qui annoncent la fin de l'humain et l'avènement du posthumain. Elle a le visage, l'âge, le sexe et le corps d'un cyborg du troisième millénaire : son enveloppe est neutre. Elle est hélas ce vers quoi l'Homme va (Michel Onfray, blog personnel, 23 juillet 2019);

- Outre qu'elle affiche son Asperger comme un titre de noblesse, son visage terriblement angoissant semble dire : si vous ne le faites pas pour la planète, faites-le au moins pour moi (Pascal Bruckner, Le Figaro, 10 avril 2019);

- Mais elle a aussi comme deuxième prénom, Tintin, dont elle a le physique et l'angélisme (François Clémenceau, JDD, 2 mars 2019).

40 Ces qualifications physiques s'inscrivent dans un contexte de dépréciation de l'adversaire érigé en figure qui concentre des attaques de tous ordres, cristallisant alors une série d'attaques qui se répercutent sur l'apparence corporelle. 


\section{De la confusion entre construction critique et destruction dépréciative}

41 Cette étude pourrait être complétée par la constitution d'un second corpus, formé de tweets ou de commentaires Facebook, tant l'univers numérique regorge de productions énonciatives qui mériteraient une étude à part entière, pour ce qui est des opérations de dénigrement de Thunberg. Nous pouvons cependant remarquer un certain nombre d'éléments centraux, en croisant nos différentes analyses.

Tout d'abord, les arguments concernant l'écologie sont absents dans les critiques à l'égard de Greta Thunberg. Cela s'explique par une structure textuelle que l'on retrouve dans un grand nombre de publications: les auteurs attaquent la militante avant d'expliquer ce que serait pour eux une politique écologique « sérieuse » ou « réaliste ». Une fois l'adversaire ainsi déconstruite, sans lui opposer d'argument sur le fond, il devient alors possible de déployer son propre argumentaire sans avoir besoin de le justifier ou de le défendre.

43 Deuxième régularité remarquable : à travers Thunberg, c'est le militantisme qui est attaqué, dans la mesure où sa forme même ne permettrait pas de réfléchir sereinement au changement climatique (si tant est qu'il existât pour certains auteurs), et notamment les « punchlines » et slogans employés par la militante. C'est faire peu de cas de la dimension d'innovation sociale contenue dans les slogans (Colla 2013) ainsi que leur utilité dans la diffusion d'idées dans le champ public et politique (Veg 2016), leurs qualités communicationnelles propres à diffuser des messages importants lors de mouvements sociaux (Denton 1980), et leurs capacités à pouvoir contribuer de manière effective à des changements sociaux, comme l'ont montré les travaux sur la décroissance, notamment (Demaria, Schneider, Sekulova et Martinez-Allier $2013: 210$ ) :

La décroissance constitue un exemple de science inspirée par l'activisme, où un slogan activiste se consolide progressivement en un concept qui peut être analysé et discuté dans la sphère académique. [...] La diversité maintient une sorte de tension qui stimule les débats et les échanges constructifs, offrant ainsi un avantage pour un progrès continuel aux niveaux théorique et pratique [notre traduction].

Derrière cette méfiance pour les slogans se dissimule un soupçon de manipulation qui emprunte aux logiques complotistes décrites par Loïc Nicolas : ainsi, « des signes et des propriétés se retrouvent concaténées en un discours réductionniste et simple, au service d'une "rhétorique de la facilité" " (Nicolas 2014).

Enfin, cette rhétorique de la facilité s'associe à une rhétorique de la saleté, dans la mesure où Thunberg représente une figure qui centralise bon nombre d'attaques concernant des groupes sociaux qui ne sont pas en position d'exercer un pouvoir sur le monde ou sur le cours des choses (Watts 1991: 60-62). A elle seule, Greta peut représenter :

- des personnes en situation de handicap et autistes Asperger notamment, dans ce cas précis ;

- des enfants, de par son apparence juvénile abondamment soulignée et le fait qu'elle ne soit pas majeure;

- des jeunes filles, à qui l'on reprocherait de ne pas être à l'école au lieu de manifester le vendredi à propos d'un sujet dont elles ne comprennent sans doute pas l'infinie complexité ;

- des femmes, dangereuses ou incompréhensibles puisque non désirables (les remarques sur le corps juvénile de Greta Thunberg le soulignent), en proie à des situations de possession quasi-mystique (donc irrationnelles), et suscitant des émotions au sein des foules sans 
donner l'impression d'en communiquer (alors même que c'est ce que l'on pourrait « attendre d'une femme »).

Pour cette raison, Thunberg ne représente pas uniquement le combat du changement climatique aux yeux de ses détracteurs; au-delà de la question écologique, du modèle de consommation et de la nécessité d'agir pour contrer les effets du changement climatique, il y a le fait qu'il s'agit d'une jeune femme (certes entourée par une famille et une équipe qui maîtrise les méthodes de communication contemporaine), qui s'empare de questions politiques avec combativité, constance et ténacité - des qualités que l'on préfèrerait voir chez les hommes, ou bien chez des femmes certes jeunes, comme Malala Yousafzai, mais qui demeurent dans des pays culturellement éloignés des démocraties occidentales. Pour ces raisons, la question des critiques à l'égard de Greta Thunberg ne saurait être séparée des questions de genre et de société.

\section{BIBLIOGRAPHY}

Amossy, Ruth. 2011. « Des sciences du langage aux sciences sociales : l'argumentation dans le discours ", A contrario $16: 2,10-25$

Amossy, Ruth. 2015. «Quelle vocation empirique pour l'argumentation dans le discours? ", Argumentation et Analyse du Discours [En ligne] 15, consulté le 5 août 2019. http://

journals.openedition.org/aad/2059

Amossy, Ruth. 2017. « Le préconstruit dans les débats. La question des argumentaires ", Amossy, Ruth (éd). Le préconstruit. Approche pluridisciplinaire (Paris : Classiques Garnier), 83-101

Baker, Paul. 2006. Using corpora in discourse analysis (Londres : Continuum)

Bendinelli, Marion. 2011. « Anglais de spécialité et logométrie. L'exemple des débats présidentiels américains ", Asp 60, 103-123

Bischofsberger, Marco. 2002. «Quel constructivisme pour la linguistique cognitive ? , Bouquet, Simon \& François Rastier (éds). Introduction aux sciences de la culture (Paris : PUF), 157-174

Chilton, Paul. 2005. « Discourse space theory : geometry, brain and shifting viewpoints », Annual review of cognitive linguistics 3, 78-116

Colla, Elliott. 2013. «In praise of insult: slogan genres, slogan repertoires and innovation », Review of Middle East Studies 47: 1, 37-48

Dahlberg, Lincoln. 2013. « Discourse theory as critical media politics? Five questions », Dahlberg, Lincoln \& Sean Phelan (éds.). Discourse theory and critical media politics (Londres : Palgrave Macmillan), 41-63

Demaria, Federico, François Schneider, Filka Sekulova \& Joan Martinez-Allier. 2013. « What is degrowth? From an activist slogan to a social movement », Environmental Values 22: 2, 191-215

Denton, Robert. 1980. "The rhetorical functions of slogans: classifications and characteristics ", Communication Quarterly 28: 2, 10-18

Argumentation et Analyse du Discours, 25 | 2020 
Doury, Marianne \& Christian Plantin. 2015. «Une approche langagière et interactionnelle de l'argumentation ", Argumentation et Analyse du Discours [En ligne] 15, consulté le 5 août 2019. http://journals.openedition.org/aad/2006

Ducrot, Oswald. 1980. « Analyses pragmatiques », Communications $32: 1,11-60$

Fairclough, Norman. 1992. Discourse and social change (Cambridge: Polity Press)

Frigon, Sylvie \& Michèle Kérisit. 2000. Du corps des femmes : contrôles, surveillances et résistances (Ottawa : U. of Ottawa Press)

Garric, Nathalie \& Valérie Capdevielle-Mougnibas. 2009. « La variation comme principe d'exploration de corpus : intérêts et limites de l'analyse lexicométrique interdisciplinaire pour l'étude de discours ", Corpus 8, 105-128

Howarth, David. 2000. Discourse (Buckingham: Open University Press)

Marchand, Pascal. 1998. L'analyse du discours assistée par ordinateur (Paris : A. Colin)

Martinez, William. 2012. « Au-delà de la cooccurrence binaire... Poly-cooccurrences et trames de cooccurrence ", Corpus 11, 191-216

Moirand, Sophie. 2007. Les discours de la presse quotidienne. Observer, analyser, comprendre (Paris : PUF)

Nathan, Dev, Govind Kelkar \& Yu Xiaogang. 1998. «Women as witches and keepers of demons: cross-cultural analysis of struggles to change gender relations », Economic and Political Weekly 33: 44, WS58-WS59

Nicolas, Loïc. 2014. «L'évidence du complot : un défi à l'argumentation. Douter de tout pour ne plus douter du tout ", Argumentation et Analyse du Discours [En ligne] 13, consulté le 5 août 2019 http://journals.openedition.org/aad/1833

Nicolas, Loïc. 2015. Discours et liberté. Contribution à l'histoire politique de la rhétorique (Paris : Classiques Garnier)

Paveau, Marie-Anne. 2006. Les prédiscours : sens, mémoire, cognition (Paris : Presses Sorbonne Nouvelle)

Pêcheux, Michel. 1975. Les vérités de la Palice (Paris : Maspéro)

Reinert, Max. 1990. «ALCESTE - une méthodologie d'analyse des données textuelles et une application : Aurélia de Gérard de Nerval », Bulletin de méthodologie sociologique 26, 24-54.

Scott, Mike. 2001. "Comparing corpora and identifying key words, collocations, frequency distributions through the Wordsmith Tools suite of computer programs », Ghadessy, Mohsen, Henry, Alex \& Robert L. Roseberry (éds.). Small corpus studies and ELT. Theory and practice (Amsterdam: John Benjamins), 47-70

Stockinger, Peter. 2001. Traitement et contrôle de l'information (Paris : Hermès)

Tileagă, Cristian. 2007. « Ideologies of moral exclusion: A critical discursive reframing of depersonalization, delegitimization and dehumanization ", British Journal of Social Psychology 46: 4, 717-737

Tournier, Maurice. 1985. « Texte 'propagandiste' et cooccurrences. Hypothèses et méthodes pour l'étude de la sloganisation », Mots. Les langages du politique 11, 155-187

Veg, Sebastian. 2016. «Creating a textual public space: slogans and texts from Hong Kong's Umbrella movement », The Journal of Asian Studies 75: 3, 673-702 
Wagener, Albin. 2017. « Démocratie et 'coup de poker' : analyse discursive critique d'éditoriaux hostiles au référendum grec de 2015 », Le discours et la langue 9: 1, 183-201

Wagener, Albin. 2019a. Discours et système : théorie systémique du discours et analyse des représentations (Bruxelles: P. Lang)

Wagener, Albin. 2019b. « Pragmatique discursive du témoignage numérique : sexisme ordinaire dans le tumblr 'Payetafac' ", Interfaces numériques 8: 1

Watts, Richard. 1991. Power in family discourse (New York: Mouton de Gruyter)

\section{NOTES}

1. La République en Marche.

2. Site web francophone consacré à la santé et au bien-être, selon Wikipedia https://fr.wikipedia.org/wiki/Doctissimo.

3. Il s'agit d'une enquête concernant les liens entre les parents de G. Thunberg et Ingmar Rentzhog, cofondateur de la start-up We Don't Have Time, réseau social prévu pour lutter contre le réchauffement climatique, et également président du conseil du think tank Global Utmaning, qui dispose de liens avec le programme du forum de Davos Global Shapers. Dans ce cadre, la présence du réseau Rentzhog, notamment auprès de la mère de Greta, a pu jeter le discrédit sur la spontanéité supposée du combat de la jeune militante suédoise.

4. https://reporterre.net/Le-capitalisme-vert-utilise-Greta-Thunberg

5. Les mots-clés utilisés ont été simplement « critique Greta Thunberg ».

6. Selon Laks, l'adéquation de situation constitue un sentiment de pertinence cognitive entre la perception des paramètres d'un environnement et la réaction ou réponse à ces paramètres.

7. IRAMUTEQ: Interface de $\mathrm{R}$ pour les Analyses Multidimensionnelles de Textes et de Questionnaires : http://www.iramuteq.org. Ce logiciel est basé sur la méthode ALCESTE (Reinert 1990).

8. Il s'agit bien ici de l'interprétation construite par le locuteur, selon la théorie décrite par Chilton.

9. Sites d'information disposant exclusivement d'un déploiement numérique.

10. La méthode Reinert est issue de l'analyse factorielle de correspondances (cf. notre note 11) et regroupe les ensembles lexicaux par thématiques générales du corpus : elle permet de mettre en lumière l'ordre interne d'un discours, en découpant les textes en segments, regroupés en classes en fonction des mots qui les composent. Plus de détails sur http://iramuteq.org.

11. L'analyse factorielle de correspondances (AFC) transforme les données en statistiques et les organise en un graphique bidimensionnel, qui expose les relations lexicales entre les données ; cela permet de voir la différence entre classes et groupes de mot, afin de lier et hiérarchiser les informations des textes. Plus de détails sur http://iramuteq.org.

\section{ABSTRACTS}

Greta Thunberg, the champion of the school strike for climate, is well-known for her declarations at the COP24 or in front of various assemblies and parliaments. From the moment a member of 
parliament invited her to speak at the French National Assembly on Friday, July 23rd, 2019, with other French climatologists among whom Valérie Masson-Delmotte, many people have publicly produced negative comments and sceptical discourses about her. Such utterances have been published in French speaking media such as newspapers, magazines, pureplayers and blogs, to name but a few. In this context, I propose to examine how the opponents to the Swedish activist have structured their viewpoints within the discourses they produce, to study their opinions and counter-discourses, and to understand the argumentative structures underlying them.

Présentée comme l'égérie de la grève de l'école pour le climat, Greta Thunberg s'est fait connaître à travers ses déclarations à la COP24 ou devant des assemblées, parlementaires ou non. En France, l'annonce de sa venue le 23 juillet 2019 à l'Assemblée Nationale, sur invitation d'un député et accompagnée notamment de la climatologue Valérie Masson-Delmotte, a polarisé à l'égard de la militante plusieurs critiques, commentaires et discours sceptiques. Dans les productions numériques médiatiques francophones (journaux, magazines, pureplayers, blogs, etc.), des auteurs ont décidé d'afficher publiquement leurs critiques. C'est dans ce contexte que nous proposons d'observer la manière dont les détracteurs de la militante suédoise argumentent au sein des discours qu'ils mobilisent et produisent, d'analyser les critiques qu'ils émettent, et de comprendre les structures argumentatives qui les sous-tendent.

\section{INDEX}

Mots-clés: argumentation, climat, discours, écologie, Greta Thunberg, Iramuteq

Keywords: argumentation, climate, discourse, ecology, Greta Thunberg, Iramuteq

\section{AUTHOR}

\section{ALBIN WAGENER}

Université Rennes 2 ; INALCO 